\title{
25 Research Suare \\ Interleukin-17 is Involved in Neuropathic Pain and Spinal Synapse Plasticity on Mice
}

\author{
Jia-Lu Sun \\ Fudan University \\ Wen-Jing Dai \\ Fudan University \\ Xin-Yuan Shen \\ Fudan University \\ Yu-Qiu Zhang \\ Fudan University \\ Ning Lü ( $\square$ ninglu@fudan.edu.cn ) \\ Fudan University
}

\section{Research Article}

Keywords: interleukin-17, spared nerve injury, spinal LTP, astrocyte, mice

Posted Date: June 24th, 2021

DOI: https://doi.org/10.21203/rs.3.rs-512458/v1

License: (c) (i) This work is licensed under a Creative Commons Attribution 4.0 International License. Read Full License 


\section{Abstract}

Background: Neuropathic pain seriously affects people's life, but its mechanism is not clear. Interleukin-17 (IL-17) is a proinflammation cytokine and involved in pain regulation. Our previous study found that IL-17 markedly enhanced the excitatory activity of spinal dorsal neurons in mice spinal slices. The present study attempts to explore if IL-17 contributes to neuropathic pain and spinal synapse plasticity.

Methods $\llbracket A$ model of spared nerve injury (SNI) was established in C57BL/6J mice and IL-17a mutant mice. The pain-like behaviors was tested, and the expression of IL-17 and its receptor, IL-17RA, was detected. C-fiber evoked field potentials were recorded in vivo.

Results: In the spinal dorsal horn, IL-17 predominantly expressed in the superficial spinal astrocytes and IL-17RA expressed mostly in neurons and slightly in astrocytes. The SNl-induced static and dynamic allodynia was significantly prevented by pretreatment of neutralizing IL-17 antibody (intrathecal injection, $2 \mu \mathrm{g} / 10 \mu \mathrm{L}$ ) and attenuated in IL-17a mutant mice. Post-treatment of IL-17 neutralizing antibody also partially relieved the established mechanical allodynia. Moreover, spinal long-term potentiation (LTP) of C-fiber evoked field potentials, a substrate for central sensitization, was suppressed by IL-17 neutralizing antibody. Intrathecal injection of IL-17 recombinant protein $(0.2 \mu \mathrm{g} / 10 \mu \mathrm{L})$ mimicked the mechanical allodynia and facilitated the spinal LTP.

Conclusions: These data implied that IL-17 in the spinal cord played a crucial role in neuropathic pain and central sensitization.

\section{Introduction}

Neuropathic pain is one of the serious chronic pain, induced by infection, nerve trauma, ischemia, metabolic disease, or chemotherapy, which is typically characterized by allodynia, hyperalgesia, burning pain and paresthesia $[12,18,28]$. Many patients are suffering from the refractory pain with current treatments, duo to the underlying mechanisms is still incompletely understood.

Although neuropathic pain is an expression of the neural sensitization in the central nervous system (CNS), numerous data have indicated that the astrocytes and cytokines in the CNS play a key role in the mediation of neuropathic pain $[11,13,16]$. Astrocytes are abundant in the CNS, and never regarded as only support cells. Recent reports show that astrocytes have multiple active roles in acute and chronic pain. Furthermore, in both human and animal studies, astrocytes are activated in pathological pain to produce proinflammatory cytokines, such as TNF-a, IL-1 3 , and IL-18 which act as neuromodulators and regulate pain via neuron-glial or glia-glia interactions $[3,20,21]$.

In immune system IL-17 is came from Th17 cells. Within the IL-17 family, IL-17A and IL-17F are the central informs to play important role as proinflammatory cytokine against extracellular micro-organisms and in the pathogenesis of different autoimmune diseases [15, 29]. IL-17A was first described in human peripheral blood [26]. Serum levels of IL-17 were significantly upregulated in leprosy neuropathy patients 
with neural pain than without pain [1]. IL-17 receptor, IL-17RA, expression is obviously enhanced in the CNS of mice with experimental autoimmune encephalomyelitis (EAE) compared to healthy mice [7]. In vitro, astrocytes and microglia express IL-17 receptor, and IL-17A administration induced significant increase the secretion of chemokine, such as MCP-1, MCP-5, MIP-2 and KC [14]. In a mouse model of bone cancer pain (BCP), it is shown that numerous $T$ cells infiltrated into the spinal cord following sarcoma cell implantation with enhanced BCP and IL-17/IL-17A were increased in blood and the spinal cord. BCP was decreased after the intrathecal injection of IL-17/IL-17A neutralizing antibodies [14].

Injection of recombinant IL-17 of murine into the hind paw, the sciatic nerve or the knee joint of mice induced mechanical or thermal hyperalgesia [10]. After injection of complete Freund's adjuvant (CFA) into the paw IL-17 is increased in astrocytes, and IL-17RA is upregulated in neurons expressed n-methylDAspartate receptor (NMDAR) [22]. In the spinal cord, IL-17 is mainly produced by astrocytes, facilitates the phosphorylation and the trafficking of NMDARs to sensitize pain [7]. The administration of IL-17A into the normal knee joint of anaesthetized rats enhanced the responses of $C$ fibers to noxious mechanical stimuli, and a high concentration also facilitated weakly Aठ fibers [25]. IL-17 upregulated tetrodotoxin (TTX)-resistant sodium channels and increased the generation of action potentials in dorsal root ganglion (DRG) neurons [9]. It is known that spinal long term potentiation (LTP) is considered as the mechanism of chronic pain, in this study we would explore the role of spinal IL-17 in neuropathic pain and the spinal synapse plasticity.

\section{Methods}

\subsection{Animals}

Male C57BL/6J mice (eight weeks, 25-30 g) were purchased from Shanghai Experimental Animal Center of the Chinese Academy of Sciences. IL-17a mutant mouse were supplied by Jackson Laboratory (No. 016879, USA). Five mice were housed in every plastic cage with food and water available ad libitum. The artificial light was provided in a 12:12 h (light/dark) cycle from 7 AM and room temperature was kept at $22 \pm 1^{\circ} \mathrm{C}$. All animals were sacrificed by an overdose of urethane $(25 \%, 2.5 \mathrm{~g} / \mathrm{kg}$, i.p.) after the experiments. These experiment protocols were approved by the Fudan University Animal Care and Use Committee, and were conducted in accordance with the policies issued by the International Association for the Study of Pain (IASP).

\subsection{Spared nerve injury (SNI)}

All mice were anesthetized with $2 \%$ isoflurane and the incision was made at left mid-thigh level as described by Bourquin AF et al. [5]. Two of the three branches of the sciatic nerve (the tibial and common peroneal nerve) were ligated and transected together, while the sural nerve is left intact. After a tight ligation of both nerves was performed, a 1-2 mm section of the two nerves was removed. Muscle and skin were closed in two distinct layers with silk $6-0$ suture. The sham procedure was consisted of the same surgery without ligation and transection of the nerves. 


\section{3. von Frey test}

A series of von Frey filaments $(0.16,0.4,0.6,1.0,1.4,2.0 \mathrm{~g}$, Stoelting, USA) were used on ipsilateral hind paws to assess the mechanical sensitivity as described previously [8]. Mice were habituated to the testing environment for $2 \mathrm{~h}$ every day at 2-3 days before the behavioral test. They were confined under the $8 \mathrm{~cm}$ $\times 8 \mathrm{~cm} \times 4 \mathrm{~cm}$ plexiglass boxes placed on an elevated iron wires floor with $2 \mathrm{~mm}$ grids. In ascending order of force, an appropriate von Frey filament was applied on the plantar surface of the hind paw. Different filament was applied $2 \mathrm{~s} \times 5$ times between every $15 \mathrm{~s}$. The mechanical threshold was defined as paw withdrawal threshold (PWT), which was the lowest force produced 3 withdraw responses out of 5 applications.

\subsection{Allodynia Score}

As described by Cheng et al. [6], dynamic mechanical hypersensitivity was tested by light stroking from heel to toe (velocity: about $2 \mathrm{~cm} / \mathrm{s}$ ) along the external lateral side of the injured hind paw using a trimmed blunt paintbrush (the width of tip: $1 \mathrm{~mm}$, the length of tip: $6 \mathrm{~mm}$ ). The allodynia scores were graded according to the following 4-point scale: 0 , a very fast movement, lifting the stimulated paw for less than $1 \mathrm{~s} ; 1$, sustained lifting (more than $2 \mathrm{~s}$ ) toward the body or a single gentle flinching of the stimulated paw; 2 , one strong lateral paw lift, above the level of the body or a startle-like jump; 3 , multiple flinching responses or licking of the affected paw. We repeated the stimulation three times at $30 \mathrm{~s}$ intervals and obtained an average score for each mouse.

\subsection{Drug administration}

Mice were briefly anesthetized with 1-2\% isoflurane. For lumbar puncture injection, rabbit anti IL-17 antibody (Sigma, PRS4887, USA), normal rabbit IgG (R\&D, AB-105-C, USA) or IL-17 recombination (R\&D, 7956-ML/CF, USA) was delivered to the cerebral spinal fluid with a 31-gauge needle between the L4 and L5 vertebrae. No more than $10 \mu \mathrm{l}$ of drug was administered over a period of $5 \mathrm{~min}$. Sterile $0.01 \mathrm{M}$ PBS was used as the vehicle control.

\subsection{Immunohistochemistry}

Under anesthesia with urethane $(25 \%, 1.5 \mathrm{~g} / \mathrm{kg}$, i.p.), the L4-L6 spinal cord was removed after transcardial perfusion with normal saline and $4 \%$ paraformaldehyde in $0.1 \mathrm{M} \mathrm{PB}$. Thereafter, the tissue was post-fixed for $2-4 \mathrm{~h}$ at $4^{\circ} \mathrm{C}$ followed by dehydration in gradient sucrose (10\%-20\%-30\%) for $24-48 \mathrm{~h}$ at $4^{\circ} \mathrm{C}$.

Transverse spinal cord sections were $35 \mu \mathrm{m}$ made by a cryostat (model 1900, Leica, Germany). After blocked by $10 \%$ normal donkey serum with $0.3 \%$ Triton $\mathrm{X}-100$ for $2 \mathrm{~h}$ at $4^{\circ} \mathrm{C}$, the sections were incubated with the primary antibodies of rabbit anti-IL-17 (1:50, Sigma-Aldrich, PRS4887, USA), rabbit anti-IL-17 RA (1:50, Santa Cruz, SC-30175, USA), goat anti-Iba-1 (1:500, Abcam, ab5076, UK), mouse anti-GFAP (1:2000, Sigma-Aldrich, G6171, USA), or mouse anti-NeuN (1:2000, Millipore, MAB377, USA) overnight at $4^{\circ} \mathrm{C}$. Then the sections were labeled with secondary antibodies of donkey anti-goat Alexa Flour 546 (1:200, Invitrogen, A-11055, USA), donkey anti-rabbit Alexa Flour 488 (1:200, Invitrogen, A-10040, USA) or donkey 
anti-mouse Alexa Flour 546 (1:200, Invitrogen, A-10036, USA) for $2 \mathrm{~h}$ at room temperature in the dark. The images were captured by a confocal laser-scanning microscope (FV1000; Olympus, Tokyo, Japan).

\subsection{Recording of spinal LTP in vivo}

According to the previous study [4], mice were anesthetized with urethane $(1.5 \mathrm{~g} / \mathrm{kg}$, i.p.). For exposing the lumbar enlargement, a laminectomy was performed at vertebrae T13 to L1. A PE-5 tube was inserted into the gap of $L 4$ and $L 5$ vertebrae and extended to the cavum subarachnoidale space near the lumbar enlargement. Approximately $2 \mu$ sterile normal saline (NS) was filled in the catheter, and the drugs or vehicle $(10 \mu \mathrm{l})$ were applied in 1 min followed by $2 \mu \mathrm{lNS}$. The left sciatic nerve was separated free for bipolar electrical stimuli. A feedback-controlled heating blanket was used for keeping the colorectal temperature at $37 \pm 0.5^{\circ} \mathrm{C}$.

The field potentials were recorded with glass microelectrodes (3-5 $\mathrm{M} \Omega$ impedance) in 100-300 $\mu \mathrm{m}$ from the surface of the L4-L5 spinal cord segments. A single rectangular pulse ( $2 \times \mathrm{C}$-fiber threshold, $0.5 \mathrm{~ms}, 60$ s interval) was applied on the ipsilateral sciatic nerve. Followed the stable recordings more than $30 \mathrm{~min}$, spinal LTP was induced by the conditioning tetanic stimulation ( 4 x C-fiber threshold, $0.5 \mathrm{~ms}, 100 \mathrm{~Hz}, 4$ trains of $1 \mathrm{~s}$ duration, $10 \mathrm{~s}$ interval) delivered to the sciatic nerve. As a control, the sham group was not applied with conditioning tetanic stimulation. The signals were amplified by a microelectrode AC amplifier (A-M System, USA), and converted into a digital signal by CED systems (A/D converter Micro $1401 \mathrm{mk} \bigotimes, \mathrm{UK})$.

The responses of 10 consecutive test stimuli calculated off-line by Spike 2 version 6 were averaged and normalized to mean amplitudes of C-fiber evoked field potentials in the first 30-min.

\subsection{Statistics}

All data were expressed as mean \pm SEM and tested using Student's $t$ test, one-way or two-way ANOVA followed by post hoc Student-Newmann-Keuls test. The criterion for statistical significance was $P<0.05$.

\section{Results}

\subsection{IL-17 and IL-17R were expressed in the spinal cord dorsal horn.}

To determine the spatial distribution of IL-17 and IL-17R, we performed double immunofluorescent staining for IL-17/IL-17RA and NeuN (neuronal markers), Iba-1 (a microglial marker), or GFAP (an astrocytic marker) on sections of the L4-L6 spinal cord. Moderate IL-17/IL-17R immunoreactivities were found in the superficial layers I-II of the spinal dorsal horn in normal mice. IL-17 was co-localized with GFAP and IL-17R was mainly co-expressed with NeuN or slightly with GFAP. Both IL-17 and IL-17RA labeled cells were rarely in Iba-1 labeled microglia (Fig. 1-2).

\subsection{IL-17 was involved in SNI-induced neuropathic pain.}


Following SNI, mechanical allodynia was induced in mice lasting four weeks at least. The static PWTs were dramatically decreased at $4 \mathrm{~d}$ after surgery on ipsilateral hind paw compared with that of sham mice (Fig. 3A; Two-way RM ANOVA, treatments: $F_{3,24}=88.36, P<0.001$ ), while the dynamic allodynia scores were obviously increased at the same time (Fig. 3B; Two-way RM ANOVA, treatments: $F_{3,22}=$ $121.2, P<0.001)$.

To address whether spinal IL-17 is involved in SNI, we examined the influence of blocking IL-17 on SNIinduced allodynia. IL-17 neutralizing antibody (IL-17AB, $2 \mu \mathrm{g} / 10 \mu \mathrm{L}$ ) was delivered intrathecally $1 \mathrm{~h}$ before SNI, PWTs were significantly increased compared with IgG at 4, 7, 10 and 14 days. (Fig. 3C; Two-way RM ANOVA, treatments: $\left.F_{3,16}=47.02, P<0.001\right)$ and the allodynia scores were also decreased at $4,7,10$ and 14 days (Fig. 3D; Two-way RM ANOVA, treatments: $F_{3,16}=48.99, P<0.001$ ). Similarly, mechanical allodynia induced by SNI was delayed and attenuated in IL-17a mutant mice compared with wide-type mice (Fig. 3E; Two-way RM ANOVA, treatments: $F_{1,13}=9.08, P=0.01$. Figure 3F; Two-way RM ANOVA, treatments: $\left.F_{1,14}=20.77, P<0.001\right)$. Moreover, we revealed that intrathecal injection of IL-17AB $(2 \mu \mathrm{g} / 10$ $\mu \mathrm{L}$ ) also relieved the established neuropathic pain on day 10 after SNI. PWTs were significantly increased on 1 or $3 \mathrm{~h}$, and the allodynia scores were decreased respectively (Fig. 3G; Two-way RM ANOVA, treatments: $F_{1,8}=6.353, P<0.05$. Figure $3 \mathrm{H}$, Two-way RM ANOVA, treatments: $\left.F_{1,8}=7.835, P<0.05\right)$.

Next, to address whether exogenous IL-17 into the spinal dorsal horn directly evokes allodynia, IL-17 recombination $(0.2 \mu \mathrm{g} / 10 \mu \mathrm{L})$ was intrathecally injected. The mechanical allodynia was obviously induced at $1 \mathrm{~h}$ after injection and lasting 5 hours compared with vehicle (Fig. 3l; Two-way RM ANOVA, treatments: $\left.F_{1,13}=13.67, P<0.01\right)$.

\subsection{IL-17 contributed to spinal synapse plasticity.}

The previous studies showed that LTP of C-fiber evoked potentials in the spinal cord was regarded as a substrate for central sensitization of the pain pathway which amplifies nociceptive input and results in hyperalgesia [17, 27]. In this study, the spinal LTP dose-relatedly alleviated by IL-17 antibody $(0.2 \mu \mathrm{g}$ or 2 $\mu \mathrm{g} / 10 \mu \mathrm{l}$ ) intrathecally delivered by the PE-5 tube $1 \mathrm{~h}$ earlier than tetanic sciatic stimulation (TSS) compared to normal rabbit IgG (Fig. 4A; Two-way RM ANOVA, treatments: $F_{2,12}=11.11, P<0.01$ ).

To confirm whether IL-17 was involved in the induction of the spinal LTP, the IL-17 recombinant protein was applied intrathecally $1 \mathrm{~h}$ before TSS. The IL-17 recombination $(0.2 \mu \mathrm{g})$ facilitated the weak LTP induced by low TSS ( 4 x C-fiber threshold, $100 \mathrm{~Hz}, 0.5 \mathrm{~ms}, 2$ trains of $1 \mathrm{~s}$ duration at $10 \mathrm{~s}$ interval; Fig. 4B; Two-way RM ANOVA, treatments: $\left.\mathrm{F}_{2,264}=78.77, P<0.001\right)$.

\section{Discussion}

The present study revealed an involvement of IL-17 in neuropathic pain and spinal synapse plasticity. The static and dynamic allodynia induced by SNI was significantly prevented by intrathecal injection of IL$17 \mathrm{AB}$ before SNI. As a support, SNI-induced mechanical allodynia was robustly delayed and reduced in IL- 
17a mutant mice. Blocking of spinal IL-17 also decreased the established mechanical allodynia in SNI mice. Spinal LTP was significantly suppressed and blocked by low dose and high dose of IL-17 AB, respectively. Mouse recombinant protein of IL-17 mimicked the mechanical allodynia and sensitized the spinal LTP. Together with that IL-17 predominantly expressed in the spinal astrocytes and its receptor, IL17RA, mostly expressed in neurons and slightly in astrocytes, these data suggest that spinal IL-17 from astrocytes might modulate dorsal horn neural plasticity directly by IL-17RA expressing neurons, or indirectly via IL-17RA expressing spinal glia, thereby contributing to the neuropathic pain.

IL-17 acted as a potent inflammatory cytokine has multiple activities, one of which is to induce the expression of other proinflammatory cytokines (such as IL-6 and TNFa), chemokines (such as MCP-1, MIP-2 and KC) and matrix metalloproteases (MMPs). Expression of IL-17 has also been detected in the blood and target tissues of patients with various autoimmune diseases, including rheumatoid arthritis (RA), multiple sclerosis (MS), systemic lupus erythematous and asthma. IL-17 knock-out mice develop EAE with delayed onset and less severity. The local IL-17 concentration within the mBSA-induced arthritis was significantly enhanced. The neutralizing antibody of IL-17 relieved the pain behavior and decreased the neutrophil recruitment. Moreover, intra-articular injection of IL-17 induced hypersensitivity and neutrophil migration $[2,21,24]$. Our research also revealed that mechanical allodynia induced by SNI was ameliorated in IL-17 mutant mice. Intrathecal injection of IL-17 recombinant mimicked the pain behavior of SNI and neutralizing IL-17 antibody prevented the static and dynamic allodynia. It was reported that IL17 levels of sciatic nerve in $\mathrm{CCl}$, partial sciatic ligation (PSL) or perineural inflammation (Neuritis), were enhanced in 3 and 8 days after surgery [23]. Mice intrathecally injected with IL-17 neutralized antibody showed relieved hyperalgesia 7 days after SNL, a significant decrease of p-calmodulinkinase II and phospho-cAMP response element-binding protein in the spinal cord [31].

Li et al. first reported that IL-17-positive cells were found in the ischemic lesion region in human brain. The levels of IL-17 in the ischemic hemisphere were increased compared with the opposite normal hemispheres and peaked at 3-5 days [19]. Accordingly, we found IL-17 expressed exclusively in GFAPimmunoreactivity astrocytes in the spinal cord dorsal horn. However, IL-17RA was primarily expressed in spinal cord neurons. The expression of IL-17/IL-17R provided a cellular basis for neuron-astroglial interaction in neuropathic pain, which meant IL-17/IL-17R signaling could regulate central sensitization by neuron-glial cross talk in the CNS.

One characterization of peripheral neuropathic pain is the abnormal excitability of the nervous system. After peripheral nerve injury C-fiber input may arise spontaneously and drive peripheral and central sensitization. The activity dependent form of central sensitization is responsible for generating secondary pinprick hyperalgesia and dynamic tactile allodynia [30]. Our previous study presented that IL-17 is sufficient and required for excitatory and inhibitory synaptic transmission. Exogenous IL-17 quickly enhanced EPSCs and decreased IPSCs in the spinal cord slices from naïve mice. The other data showed that inhibitory synaptic transmission in lamina Ilo SOM + neurons was profoundly suppressed by IL-17 in mice of chemotherapy-induced neuropathic pain [20]. IL-17 specifically decreased GABA-induced currents but not glycine-induced currents. In this study, we found that IL-17 facilitated spinal LTP and pre-blocking 
IL-17 reversed the TSS-induced spinal LTP. These data indicated that IL-17 was involved in central sensitization.

\section{Conclusion}

These results suggested that IL-17 in the spinal astrocytes was involved in neuropathic pain and the synapse plasticity. It is a crucial target for the treatment of chronic pain.

\section{Abbreviations}

BCP: bone cancer pain

CFA: complete Freund's adjuvant

DRG: dorsal root ganglion

EAE: experimental autoimmune encephalomyelitis

IL-17: Interleukin-17

IL-17AB: Interleukin-17 antibody

LTP: long-term potentiation

MMPs: matrix metalloproteases

MS: multiple sclerosis

NMDAR: n-methyl-DAspartate receptor

PSL: partial sciatic ligation

PWT: paw withdrawal threshold

$\mathrm{RA}$ : rheumatoid arthritis

SNI: spared nerve injury

TSS: tetanic sciatic stimulation

TTX: tetrodotoxin

\section{Declarations}

Ethics approval and consent to participate 
These experiment protocols were approved by the Fudan University Animal Care and Use Committee, and were conducted in accordance with the policies issued by the International Association for the Study of Pain (IASP). All animals were sacrificed by an overdose of urethane $(25 \%, 2.5 \mathrm{~g} / \mathrm{kg}$, i.p.) after the experiments.

\section{Competing of Interests}

The authors declare no conflicts of interest regarding the publication of this paper.

\section{Arrive guidelines}

The study was carried out in compliance with the ARRIVE guidelines.

\section{Availability of data and materials}

The datasets used and/or analysed during the current study available from the corresponding author on reasonable request.

\section{Funding}

This work was supported by the National Natural Science Foundation of China (81571064), funds from the innovative research team of high-level local universities in Shanghai, Shanghai Municipal Science and Technology Major Project grant No. 2018SHZDZX01, and funds from Zhang Jiang Laboratory.

\section{Authors' contributions}

JS performed the behavior tests and WD performed the in vivo recording of LTP. They both analyzed and interpreted the relevant data. XSh performed the immunohistological examination. LN designed these experiments and wrote the manuscript. YZh conducted the protocol and revised the manuscript. All authors read and approved the final manuscript.

\section{Acknowledgements}

Not Applicable.

\section{References}

1. Angst DBM, Pinheiro RO, Vieira JSDS, Cobas RA, Hacker MAV, Pitta IJR, Giesel LM, Sarno EN, Jardim MR. Cytokine Levels in Neural Pain in Leprosy. Front Immunol. 2020;11:23.

2. Bettelli $E$, Oukka $M$, Kuchroo VK. T(H)-17 cells in the circle of immunity and autoimmunity. Nat Immunol. 2007;8(4):345-50.

3. Bian C, Wang ZC, Yang JL, Lu N, Zhao ZQ, Zhang YQ. Up-regulation of interleukin-23 induces persistent allodynia via CX3CL1 and interleukin-18 signaling in the rat spinal cord after tetanic sciatic stimulation. Brain Behav Immun. 2014;37:220-30. 
4. Bian C, Zhao ZQ, Zhang YQ, Lü N. Involvement of CX3CL1/CX3CR1 signaling in spinal long term potentiation. PLoS One. 2015;10(3):e0118842.

5. Bourquin AF, Süveges M, Pertin M, Gilliard N, Sardy S, Davison AC, Spahn DR, Decosterd I. Assessment and analysis of mechanical allodynia-like behavior induced by spared nerve injury (SNI) in the mouse. Pain. 2006;122(1-2):14.e1-14.

6. Cheng L, Duan B, Huang T, Zhang Y, Chen Y, Britz O, Garcia-Campmany L, Ren X, Vong L, Lowell BB, Goulding M, Wang Y, Ma Q. Identification of spinal circuits involved in touch-evoked dynamic mechanical pain. Nat Neurosci. 2017;20(6):804-814.

7. Das Sarma J, Ciric B, Marek R, Sadhukhan S, Caruso ML, Shafagh J, Fitzgerald DC, Shindler KS, Rostami A. Functional interleukin-17 receptor $A$ is expressed in central nervous system glia and upregulated in experimental autoimmune encephalomyelitis. J Neuroinflammation. 2009;6:14.

8. Dai WJ, Sun JL, Li C, Mao W, Huang YK, Zhao ZQ, Zhang YQ, Lü N. Involvement of Interleukin-10 in Analgesia of Electroacupuncture on Incision Pain. Evid Based Complement Alternat Med. 2019:8413576.

9. Ebbinghaus M, Natura G, Segond von Banchet G, Hensellek S, Böttcher M, Hoffmann B, Salah FS, Gajda M, Kamradt T, Schaible HG. Interleukin-17A is involved in mechanical hyperalgesia but not in the severity of murine antigen-induced arthritis. Sci Rep. 2017;7(1):10334.

10. Ebersberger A. The analgesic potential of cytokine neutralization with biologicals. Eur J Pharmacol. 2018;835:19-30.

11. Gao YJ, Ji RR. Targeting astrocyte signaling for chronic pain. Neurotherapeutics. 2010;7(4):482-93.

12. Grace PM, Hutchinson MR, Maier SF, Watkins LR. Pathological pain and the neuroimmune interface. Nat Rev Immunol. 2014;14(4):217-31.

13. Hung AL, Lim M, Doshi TL. Targeting cytokines for treatment of neuropathic pain. Scand J Pain. 2017;17:287-293.

14. Huo W, Liu Y, Lei Y, Zhang Y, Huang Y, Mao Y, Wang C, Sun Y, Zhang W, Ma Z, Gu X. Imbalanced spinal infiltration of Th17/Treg cells contributes to bone cancer pain via promoting microglial activation. Brain Behav Immun. 2019;79:139-151.

15. Isailovic N, Daigo K, Mantovani A, Selmi C. Interleukin-17 and innate immunity in infections and chronic inflammation. J Autoimmun. 2015;60:1-11.

16. Ji RR, Berta T, Nedergaard M. Glia and pain: is chronic pain a gliopathy? Pain. 2013;154 Suppl 1(0 1):S10-S28.

17. Ji RR, Woolf CJ. Neuronal plasticity and signal transduction in nociceptive neurons: implications for the initiation and maintenance of pathological pain. Neurobiol Dis. 2001;8(1):1-10.

18. Ji RR, Xu ZZ, Gao YJ. Emerging targets in neuroinflammation-driven chronic pain. Nat Rev Drug Discov. 2014;13(7):533-48.

19. Li GZ, Zhong D, Yang LM, Sun B, Zhong ZH, Yin YH, Cheng J, Yan BB, Li HL. Expression of interleukin17 in ischemic brain tissue. Scand J Immunol. 2005;62(5):481-6. 
20. Luo H, Liu HZ, Zhang WW, Matsuda M, Lv N, Chen G, Xu ZZ, Zhang YQ. Interleukin-17 Regulates Neuron-Glial Communications, Synaptic Transmission, and Neuropathic Pain after Chemotherapy. Cell Rep. 2019;29(8):2384-2397.e5.

21. McGeachy MJ, Bak-Jensen KS, Chen Y, Tato CM, Blumenschein W, McClanahan T, Cua DJ. TGF-beta and IL- 6 drive the production of IL-17 and IL- 10 by $T$ cells and restrain $T(\mathrm{H})-17$ cell-mediated pathology. Nat Immunol. 2007;8(12):1390-7.

22. Meng X, Zhang Y, Lao L, Saito R, Li A, Bäckman CM, Berman BM, Ren K, Wei PK, Zhang RX. Spinal interleukin-17 promotes thermal hyperalgesia and NMDA NR1 phosphorylation in an inflammatory pain rat model. Pain. 2013;154(2):294-305.

23. Noma N, Khan J, Chen IF, Markman S, Benoliel R, Hadlaq E, Imamura Y, Eliav E. Interleukin-17 levels in rat models of nerve damage and neuropathic pain. Neurosci Lett. 2011;493(3):86-91.

24. Pinto LG, Cunha TM, Vieira SM, Lemos HP, Verri WA Jr, Cunha FQ, Ferreira SH. IL-17 mediates articular hypernociception in antigen-induced arthritis in mice. Pain. 2010;148(2):247-256.

25. Richter F, Natura G, Ebbinghaus M, von Banchet GS, Hensellek S, König C, Bräuer R, Schaible HG. Interleukin-17 sensitizes joint nociceptors to mechanical stimuli and contributes to arthritic pain through neuronal interleukin-17 receptors in rodents. Arthritis Rheum. 2012;64(12):4125-34.

26. Rouvier E, Luciani MF, Mattéi MG, Denizot F, Golstein P. CTLA-8, cloned from an activated T cell, bearing AU-rich messenger RNA instability sequences, and homologous to a herpesvirus saimiri gene. J Immunol. 1993;150(12):5445-56.

27. Sandkühler J. Understanding LTP in pain pathways. Mol Pain. 2007;3:9.

28. Sun C, Zhang J, Chen L, Liu T, Xu G, Li C, Yuan W, Xu H, Su Z. IL-17 contributed to the neuropathic pain following peripheral nerve injury by promoting astrocyte proliferation and secretion of proinflammatory cytokines. Mol Med Rep. 2017;15(1):89-96.

29. Tabarkiewicz J, Pogoda K, Karczmarczyk A, Pozarowski P, Giannopoulos K. The Role of IL-17 and Th17 Lymphocytes in Autoimmune Diseases. Arch Immunol Ther Exp (Warsz). 2015;63(6):435-49.

30. Woolf CJ. Dissecting out mechanisms responsible for peripheral neuropathic pain: implications for diagnosis and therapy. Life Sci. 2004;74(21):2605-10.

31. Yao CY, Weng ZL, Zhang JC, Feng T, Lin Y, Yao S. Interleukin-17A Acts to Maintain Neuropathic Pain Through Activation of CaMKII/CREB Signaling in Spinal Neurons. Mol Neurobiol. 2016;53(6):39143926.

\section{Figures}




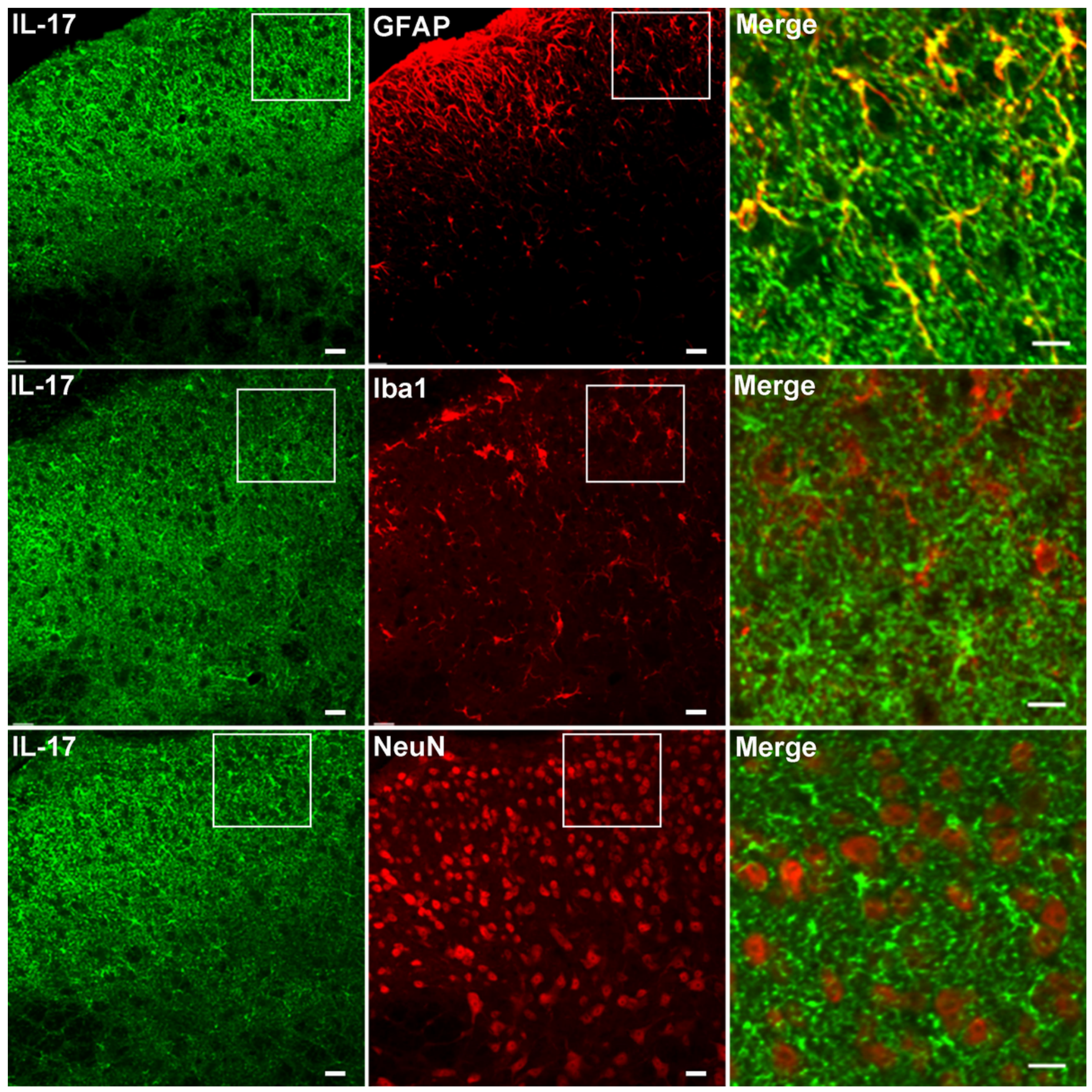

\section{Figure 1}

Expression of IL-17 in the spinal cord. Double immunofluorescence revealed that IL-17 was expressed in GFAP-labeled astrocytes, but not in Iba-1-labeled microglia and NeuN-labeled neurons in normal mice. Scale bar: $50 \mu \mathrm{m}$. 


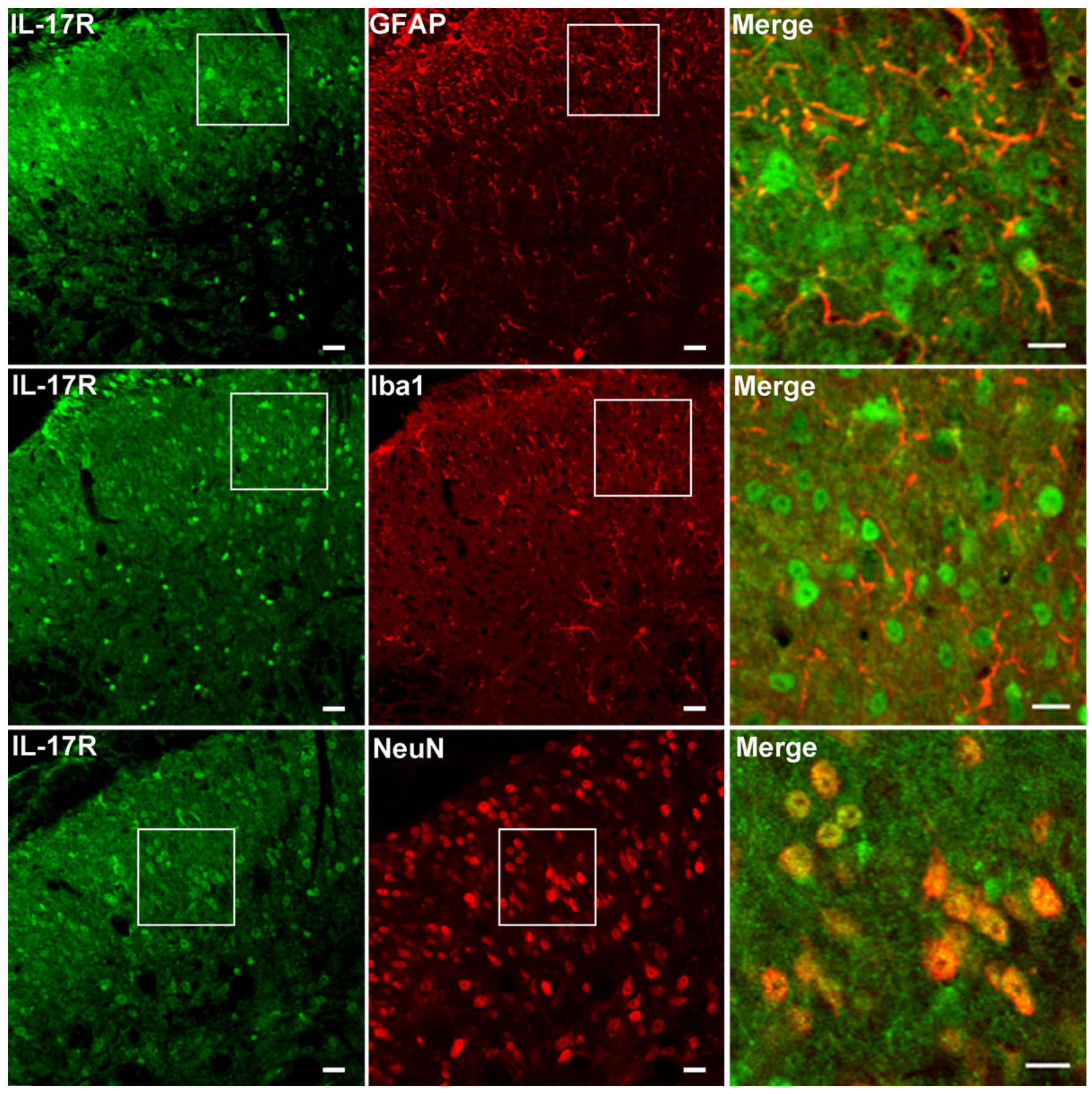

Figure 2

Expression of IL-17RA in the spinal cord. IL-17RA was expressed primarily in NeuN-labeled neurons, slightly in GFAP-labeled astrocytes, but not in Iba-1-labeled microglia. Scale bar: $50 \mu \mathrm{m}$. 
A
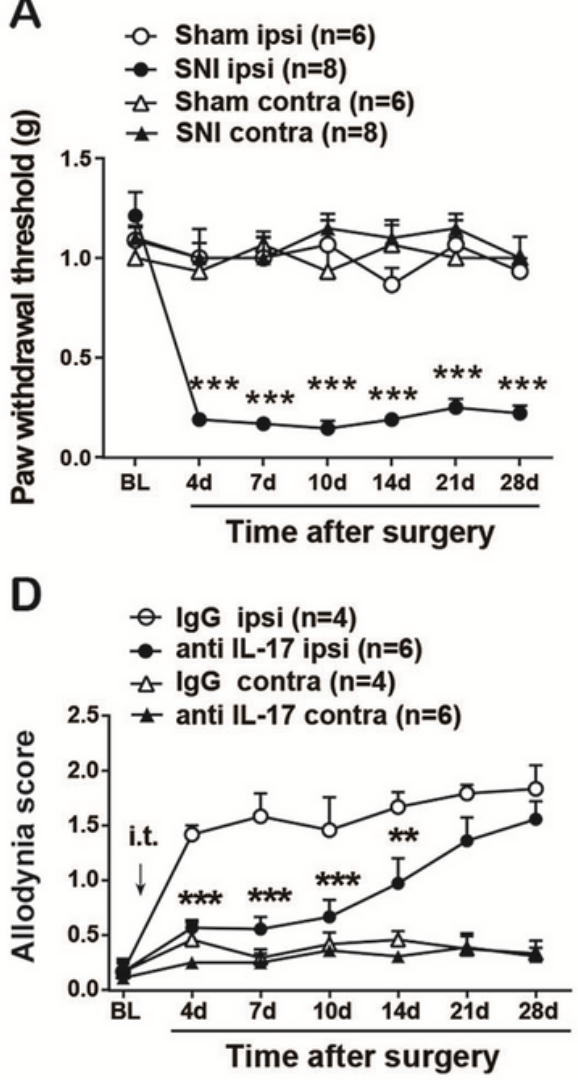

G

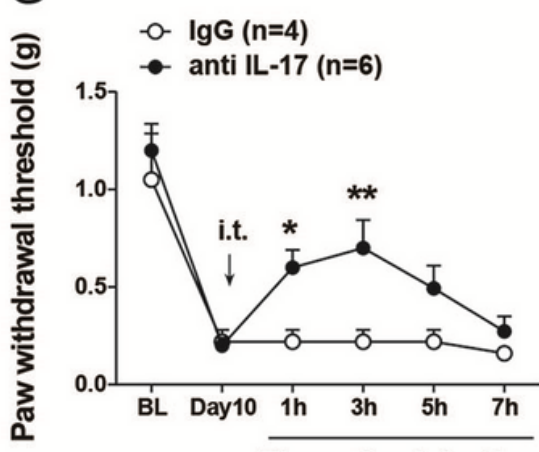

Time after injection
B

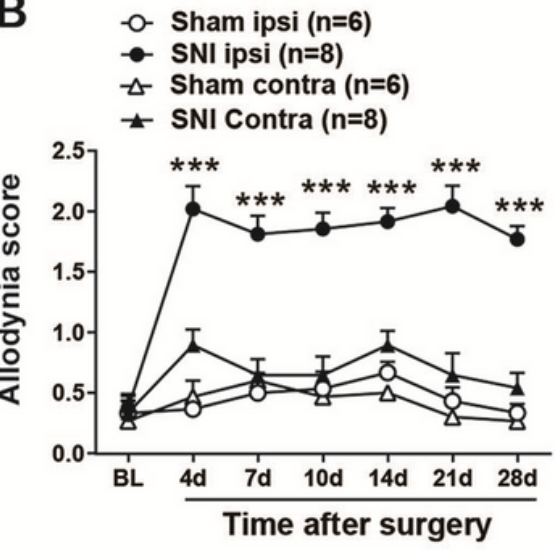

E



H

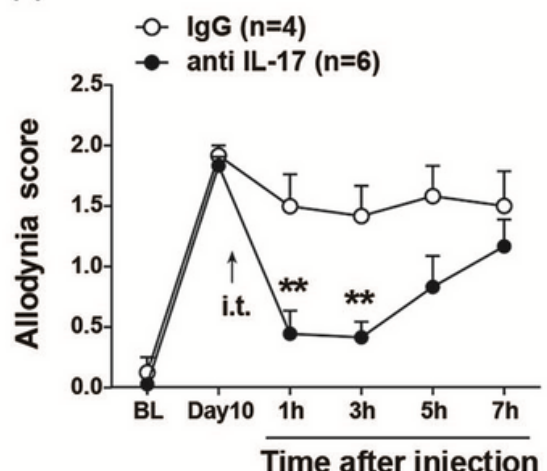

C

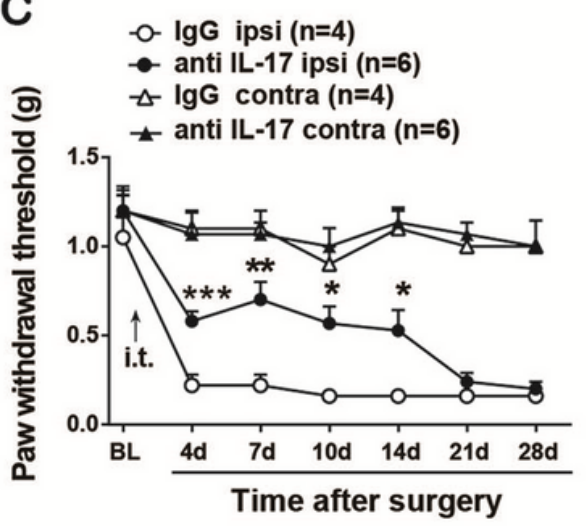

$\mathbf{F}$

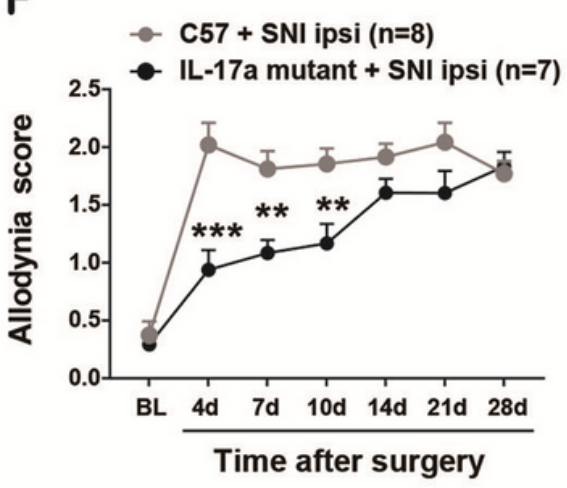

I

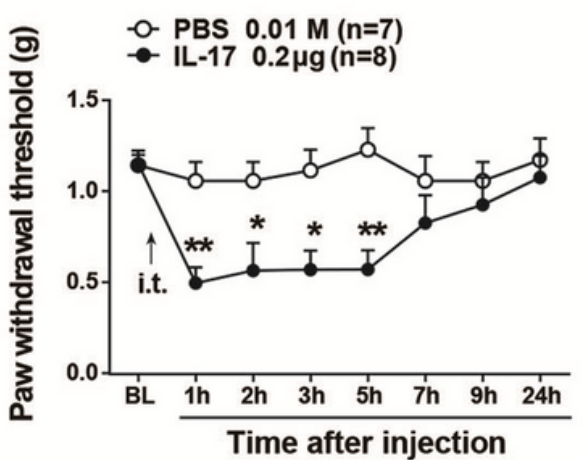

Figure 3

Involvement of IL-17 in SNI-induced neuropathic pain. a, b SNI induced static (a) and dynamic (b)

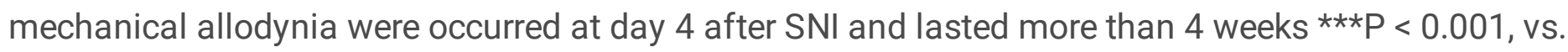
sham. c, d SNI-induced static (c) and dynamic (d) mechanical allodynia were partially prevented by pretreatment of IL-17 neutralizing antibody (IL-17AB, $2 \mu \mathrm{g} / 10 \mu \mathrm{L}$ ). IL-17AB was intrathecally injected (i.t.) one hour before SNI. * $\mathrm{P}<0.05$, ${ }^{\star \star} \mathrm{P}<0.01$, $* \star \star \mathrm{P}<0.001$, vs. IgG. e, f SNI-induced mechanical allodynia was delayed and decreased in IL-17a mutant mice. ${ }^{\star *} \mathrm{P}=0.01$ and ${ }^{* \star \star} \mathrm{P}<0.001$, vs. wide-type mice. $\mathrm{g}, \mathrm{h}$ IL-17AB $(2 \mu \mathrm{g} / 10 \mu \mathrm{L}$, i.t. $)$ reversed established mechanical allodynia at 10 days after $S N I . * P<0.05$, vs. IgG. i IL-17 recombination $(0.2 \mu \mathrm{g} / 10 \mu \mathrm{L}$, i.t.) directly induced static mechanical allodynia * $\mathrm{P}<0.05$, $*$ $P$ $<0.01$, vs. PBS. 

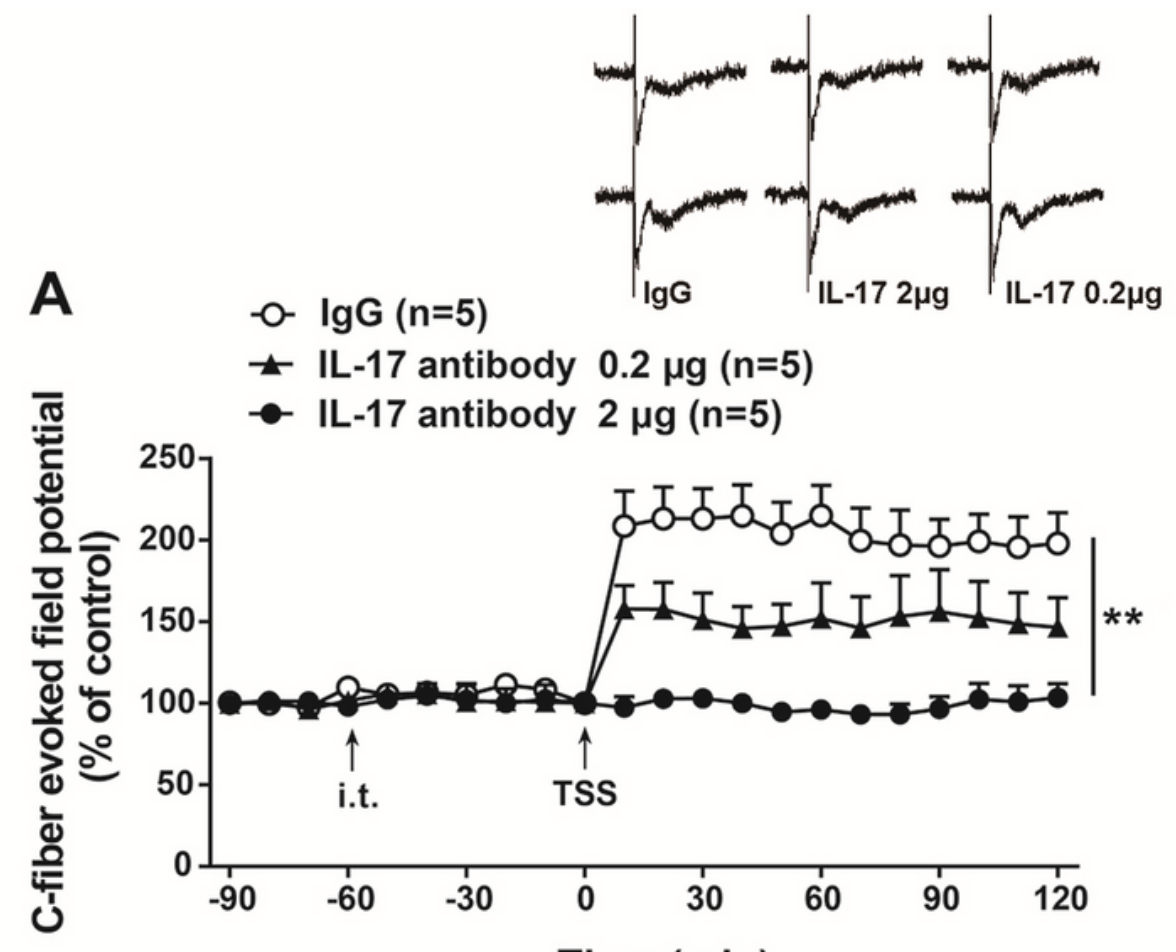

Time (min)

B

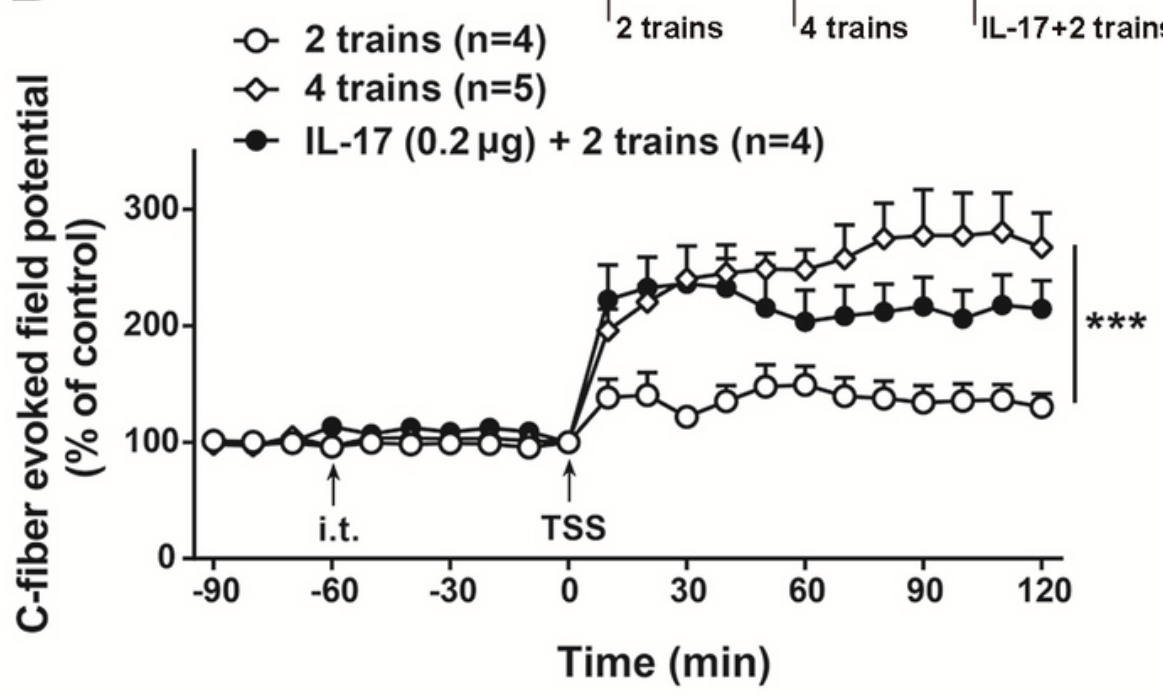

Figure 4

Involvement of IL-17 in the introduction of spinal LTP. a Four trains tetanic stimulation of the sciatic nerve (TSS)-induced spinal LTP of C-fiber evoked field potentials was completely blocked by high dose of i.t. IL17 neutralizing antibody (IL-17AB, $2 \mu \mathrm{g} / 10 \mu \mathrm{L})$ one hour before TSS. Low dose of IL-17AB $(0.2 \mu \mathrm{g} / 10 \mu \mathrm{L})$ partially blocked TSS-induced spinal LTP. b Two trains of TSS induced a weak spinal LTP compared with 
4-trains TSS. IL-17 recombination $(0.2 \mu \mathrm{g} / 10 \mu \mathrm{L}$, i.t.) facilitated 2-trains TSS-induced spinal LTP. ** $\mathrm{P}<$ $0.01, \star \star \star \mathrm{P}<0.001$, Two-way RM ANOVA. 